\title{
A Discrete Solution for the Paradox of Achilles and the Tortoise
}

\author{
Vincent Ardourel
}

Received: date / Accepted: date

\begin{abstract}
In this paper, I present a discrete solution for the paradox of Achilles and the tortoise. I argue that Achilles overtakes the tortoise after a finite number of steps of Zeno's argument if time is represented as discrete. I then answer two objections that could be made against this solution. First, I argue that the discrete solution is not an ad hoc solution. It is embedded in a discrete formulation of classical mechanics. Second, I show that the discrete solution cannot be falsified experimentally.
\end{abstract}

Keywords Achilles and the Tortoise Paradox · Discrete · Time - Discrete Mechanics . Motion · Supertasks

\section{Introduction}

The paradox of Achilles and the tortoise is one of the most famous paradoxes on motion. According to Zeno's argument, Achilles can never overtake a tortoise in a footrace if he gives him a head start. In order to pass the tortoise, Achilles must first reach the initial position of the tortoise. But during this time, the tortoise moves ahead. Achilles must then reach the new position. But, again, the tortoise reaches a new point during this time; and so on. Whenever Achilles reaches a point, the tortoise reaches a further point and Achilles never overtakes the tortoise.

It is commonly argued that the paradox can be resolved with geometric series. Suppose for example that the tortoise runs at a speed of one unit. Suppose also that Achilles runs twice as fast as the tortoise and grants him a unit length as a head start. Achilles has thus to cover the sum of lengths $1+\frac{1}{2}+\frac{1}{4}+\ldots$ in the time interval $\frac{1}{2}+\frac{1}{4}+\frac{1}{8}+\ldots$ Both geometric series converge to 2 and 1 respectively. Consequently, and contrary to Zeno's conclusion, Achilles overtakes the tortoise in a finite amount of time. This solution is however rejected by Black (1951) and Wisdom (1952). The two authors emphasize that Achilles is assumed to perform an impossible task. $\mathrm{He}$

F. Author

first address 
has to achieve a supertask, which is an infinite sequence of actions performed in a finite time. While the convergence of series shows that Achilles tends to overtake the tortoise, one cannot conclude that Achilles actually overtakes the tortoise. The debate thus focuses on the logical and physical possibilities of supertasks but there is no consensual solution yet (Benacerraf 1962, Chihara 1965, Grünbaum 1969, Thomson 1970, Alper \& Bridger 1998, Burke 2000, Laraudogoita 2006 among others).

McLaughlin \& Miller (1992) suggest another solution. They tackle Zeno's paradoxes using non-standard analysis. In this case, the race of Achilles is described as a finite number of infinitesimal steps. Non-standard analysis indeed yields a rigorous definition of infinitesimals. However, Alper \& Bridger (1997) object that this solution is based on a counterintuitive notion of finiteness. The predicate "finite" is used within the context of non-standard analysis, which does not correspond to our intuitive notion of finiteness. For example, there would be no difference between the size of a "finite" set and the size of the set of real numbers [0,1]. In his response, McLaughlin (1998) maintains his solution. The criticism of Alper \& Bridger is based on two different views of sets that have to be kept distinct. A set may be finite "internally" even if, "externally", it has infinite cardinality. However, McLaughlin recognizes that this notion of finiteness is not the traditional one. Nevertheless, it does not imply that the solution has to be rejected. One would rather reconsider our intuitive notion of finiteness. This solution of the Achilles paradox might thus depend on the acceptability of this notion of finiteness.

Another way to solve the paradox consists in denying the continuity of space and time. Even if it is not a new idea (Whitrow 1980, chapter 4), there are not many formulations of such solutions ${ }^{1}$ Van Bendegem $(1987,1995)$, referring to Forrest's work (1995), suggests a discrete treatment of space and the development of a discrete geometry in order to solve Zeno's paradoxes. In this case, contrary to the non-standard analysis based solution, the notion of finiteness is not controversial. But this solution seems to be useless for scientists. At best, it is very difficult to use discrete geometry to describe and predict physical phenomena. In this paper, I offer another kind of discrete solution. It differs from the previous one since it turns out to be usable to describe and predict physical phenomena. Besides, the solution is rather based on a discrete treatment of time than on a discrete treatment of space.

The paper is organized as follows. Section 2 is devoted to a brief presentation of the usual modern formulation of the paradox of Achilles and the tortoise. Then, in Section 3, I present the discrete solution of the paradox that, to my knowledge, has never been discussed. I show how the paradox vanishes if time becomes represented as discrete. In the remainder of the paper, I raise two possible objections and I discuss them. First, in Section 4, I tackle the objection that the discrete solution is ad hoc and cannot be a satisfying framework for physical theories. Then, in Section 5, I investigate whether the discrete solution could be falsified by an experiment.

\footnotetext{
1 Whitehead (1979, p. 35; 2000, chap. 3) defends an atomic conception of space and time. However, I do not investigate his position since it is not used to solve Zeno's paradoxes. According to him, the paradox of Achilles is already solved with the convergence of geometric series (1979, p. 69).
} 


\section{Infinite Tasks with Continuous Time}

Let us begin with the usual modern version of Zeno's argument. Time is represented by a continuous parameter $t$ defined on a real interval: $t \in I \subset \mathbb{R}$. The positions of Achilles and the tortoise are functions of time. They are written as $x_{A}(t)=v_{A} t$ and $x_{T}(t)=x_{0}+v_{T} t$ where $v_{A}$ and $v_{T}$ are respectively the constant velocities of Achilles and the tortoise, and $x_{0}$ the initial distance between them. The velocity is indeed defined as the derivative of position in respect to time $v(t)=\frac{d x}{d t}$. Since Achilles and the tortoise run at constant speed, position is an affine function of time: $x(t)=v t+x_{0}$. At the initial instant of the race, the positions of Achilles and the tortoise are $x_{A}\left(t_{0}\right)=0$ and $x_{T}\left(t_{0}\right)=x_{0}$.

According to Zeno's argument, Achilles has first to reach the initial position of the tortoise $x_{T}\left(t_{0}\right)=x_{0}$. He gets to this point at the instant $t_{1}$, which satisfies $x_{A}\left(t_{1}\right)=x_{T}\left(t_{0}\right)$, i.e $v_{A} t_{1}=x_{0}$. Hence, $t_{1}$ is defined as $t_{1}=x_{0} / v_{A}$. But during this time, the tortoise has moved ahead. Thus, after one step of Zeno's argument, Achilles is at the point $x_{A}\left(t_{1}\right)=v_{A} t_{1}$ and the tortoise at the point $x_{T}\left(t_{1}\right)=x_{0}+v_{T} t_{1}$. The tortoise still holds the first position. Achilles has now to reach the new position of the tortoise $x_{T}\left(t_{1}\right)$. He gets to this point at the instant $t_{2}$, which satisfies $x_{A}\left(t_{2}\right)=x_{T}\left(t_{1}\right)$, i.e $v_{A} t_{2}=x_{0}+v_{T} t_{1}$. This instant $t_{2}$ thus corresponds to $t_{2}=x_{0} / v_{A}+v_{T} / v_{A} t_{1}$. But, again, during this time $t_{2}$ the tortoise has moved ahead. Thus, after the second step of Zeno's argument, Achilles is at the point $x_{A}\left(t_{2}\right)=v_{A} t_{2}$ and the tortoise at the point $x_{T}\left(t_{2}\right)=x_{0}+v_{T} t_{2}$; and so on. After $n$ steps of Zeno's argument, Achilles is at the point $x_{A}\left(t_{n}\right)=v_{A} t_{n}$ and the tortoise at $x_{T}\left(t_{n}\right)=x_{0}+v_{T} t_{n}$ with the series of time $\left(t_{n}\right)_{n \in \mathbb{N}}$ defined as $x_{A}\left(t_{n}\right)=x_{T}\left(t_{n-1}\right){ }^{2}$

With these conditions, let us consider the relative position of Achilles to the position of the tortoise $x_{A / T}\left(t_{n}\right)$ at the step $n$. It corresponds to $x_{A / T}\left(t_{n}\right)=v_{r} t_{n}-x_{0}$, where $v_{r}$ is the relative speed $v_{A}-v_{T}$. This can be rewritten as $x_{A / T}\left(t_{n}\right)=-x_{0}\left(v_{T} / v_{A}\right)^{n}$. It is a negative quantity which tends to zero when the number of steps $n$ tends to infinity. Thus, after a finite number of $n$ steps, no matter how big it is, the relative distance is still strictly negative. In other words, the tortoise is still ahead of Achilles. The remaining distance becomes smaller and smaller but equals to zero only at the limit when $n$ goes to infinity. Therefore, following Black and Wisdom, Achilles overtakes the tortoise only if one endorses that Achilles performs a supertask.

\section{Achilles and the Tortoise with Discrete Time}

I propose now to follow Zeno's argument in replacing the continuous parameter $t$ by a discrete parameter $t_{k}$, where $t_{k}=h k$ with $h \in \mathbb{R}$ a discrete time step and $k \in \mathbb{N}, 0 \leq$ $k<N$. Time is now treated as discrete since it is represented by a finite set of $N$ instants. In addition, velocity is defined as $v_{k}=\frac{x_{k}-x_{k-1}}{t_{k}-t_{k-1}}$. As it will be clear in Section 4 , these definitions are guided by the recent development of a discrete formulation of classical mechanics.

\footnotetext{
2 This time series $\left(t_{n}\right)_{n \in \mathbb{N}}$ is for all $n \in \mathbb{N}^{*}, t_{n}=x_{0} / v_{A}+\left(v_{T} / v_{A}\right) t_{n-1}$ with $t_{0}=0$. It is an increasing infinite series which converges to the instant $t_{f}=x_{0} /\left(v_{A}-v_{T}\right)$. This result may be proved in rewriting the previous recurrence formula as the convergent geometric series : $t_{n}=x_{0} / v_{A} \sum_{k=0}^{n-1}\left(v_{T} / v_{A}\right)^{k}$.
} 
In our example (i) Achilles and the tortoise run at constant speed $v_{A}$ and $v_{T}$, and (ii) the initial positions of Achilles and the tortoise are respectively taken at zero and $x_{0}$. It results that $x_{A}\left(t_{k}\right)=v_{A} t_{k}$ and $x_{T}\left(t_{k}\right)=x_{0}+v_{T} t_{k}$. Accordingly, the positions of Achilles and the tortoise are also discrete since they are represented by finite sets of $N$ points ${ }^{3}$

In this section, I begin to show that Achilles overtakes the tortoise with this representation of time (Section 3.1). Then, I make clear in what sense Achilles and the tortoise can be said to be in motion although time is represented as discrete (Section $3.2)$.

\subsection{Achilles Overtakes the Tortoise}

Let us follow Zeno's argument with this discrete representation of time. Achilles starts at $x_{A}\left(t_{k_{0}}\right)=0$ and the tortoise at $x_{T}\left(t_{k_{0}}\right)=x_{0}$. Thus, in order to overtake the tortoise, Achilles has first to overtake the initial position of the tortoise ${ }^{4}$ But, during this time $t_{k_{1}}$, the tortoise has moved ahead and, after one step of Zeno's argument with discrete time, Achilles is at the point $x_{A}\left(t_{k_{1}}\right)=v_{A} t_{k_{1}}$ and the tortoise at $x_{T}\left(t_{k_{1}}\right)=$ $x_{0}+v_{T} t_{k_{1}}$. Zeno's argument continues. Achilles has now to overtake the new position of the tortoise. He overtakes this new position at the discrete time $t_{k_{2}}$. Again, during this time, the tortoise has moved ahead. He is at the new position $x_{T}\left(t_{k_{2}}\right)=x_{0}+v_{T} t_{k_{2}}$; and so on. After $n$ steps of Zeno's argument, Achilles is at the position $x_{A}\left(t_{k_{n}}\right)=v_{A} t_{k_{n}}$ and the tortoise at $x_{T}\left(t_{k_{n}}\right)=x_{0}+v_{T} t_{k_{n}}{ }^{5}$

Instead of the continuous case (Section 2), Achilles overtakes the tortoise in a finite number of steps. Let us consider again the relative position of Achilles to the tortoise at the step $n$. It is equal to $x_{A / T}\left(t_{k_{n}}\right)=v_{r} t_{k_{n}}-x_{0}$, with $v_{r}$ the relative speed. Contrary to the continuous case, there is a step $m$ of Zeno's argument where the relative position becomes a positive or nil quantity. This results from the fact that the elementary relative distance $v_{r} h$ covered in a single time step $h$ is a strictly positive finite quantity. Therefore, the discrete model satisfies the Archimedean property according to which there is an integer $k_{m}$ such as $\underbrace{v_{r} h+v_{r} h+\ldots+v_{r} h}_{k_{m}} \geq x_{0}$. Thus, after a finite number of $m$ steps, the relative position is a positive or nil quantity, which means that Achilles has overtaken the tortoise after a finite number $m$ of steps ${ }^{6}$ For example, let us consider again the case where the tortoise runs at unit velocity, Achilles runs twice as fast as the tortoise and lets him a unit length as a head start. Besides, let us take $h=10^{-5}$ for the discrete time step. Under these conditions, Achilles overtakes the tortoise after 17 steps of Zeno's argument (see Table 11).

\footnotetext{
3 Representing time as discrete is sometimes reduced to the use of natural or rational numbers (Carnap 1966, p. 88-89; Newton-Smith 1980, chapter 6). In our case, time and positions are represented as discrete although the parameters $t_{k}, x_{A}\left(t_{k}\right)$ and $x_{T}\left(t_{k}\right)$ are real numbers.

4 I discuss below in this section the use of the term "overtake" instead of "reach".

5 It can be shown that $\left(t_{k_{n}}\right)_{n \in \mathbb{N}}$ is $t_{k_{0}}=0$ and for all $n \in \mathbb{N}^{*} t_{k_{n}}=h\left\lceil\left(x_{0} /\left(h v_{A}\right)\right)+\left(v_{T} / v_{A}\right)\left(t_{k_{n-1}} / h\right)\right\rceil$, where $\lceil$.$\rceil is the ceiling function, which is defined as : \forall x \in \mathbb{R},\lceil x\rceil=\min \{n \in \mathbb{Z} \mid n \geq x\}$.

6 One can show that the order of magnitude of $m$ is $\ln \left(\frac{x_{0}}{h v_{A}}\right) / \ln \left(\frac{v_{A}}{v_{T}}\right)$.
} 
Table 1 An example of the race between Achilles and the tortoise within discrete time and with the following parameters: $v_{T}=1, v_{A}=2, x_{0}=1$ and $h=10^{-5}$. Under these conditions, Achilles overtakes the tortoise after 17 steps of Zeno's argument.

\begin{tabular}{llllll}
\hline $\begin{array}{l}\text { Step } i \text { of } \\
\text { Zeno's argument }\end{array}$ & $k_{i}$ & $\begin{array}{l}\text { instant } \\
t_{k_{i}}\end{array}$ & $\begin{array}{l}\text { Achilles } \\
x_{A}\left(t_{k_{i}}\right)\end{array}$ & $\begin{array}{l}\text { the tortoise } \\
x_{T}\left(t_{k_{i}}\right)\end{array}$ & $\begin{array}{l}\text { distance remaining } \\
\Delta_{n}\end{array}$ \\
\hline 0 & 0 & 0 & 0 & 1 & 1 \\
1 & 50,000 & 0.50000 & 1.00000 & 1.50000 & 0.50000 \\
2 & 75,000 & 0.75000 & 1.50000 & 1.75000 & 0.25000 \\
3 & 87,500 & 0.87500 & 1.75000 & 1.87500 & 0.12500 \\
4 & 93,750 & 0.93750 & 1.87500 & 1.93750 & 0.06250 \\
5 & 96,875 & 0.96875 & 1.93750 & 1.96875 & 0.03125 \\
6 & 98,438 & 0.98438 & 1.96876 & 1.98438 & 0.01562 \\
7 & 99,219 & 0.99219 & 1.98438 & 1.99219 & 0.00781 \\
8 & 99,610 & 0.99610 & 1.99220 & 1.99610 & 0.00390 \\
9 & 99,805 & 0.99805 & 1.99610 & 1.99805 & 0.00195 \\
10 & 99,903 & 0.99903 & 1.99806 & 1.99903 & 0.00097 \\
11 & 99,952 & 0.99952 & 1.99904 & 1.99952 & 0.00048 \\
12 & 99,976 & 0.99976 & 1.99952 & 1.99976 & 0.00024 \\
13 & 99,988 & 0.99988 & 1.99976 & 1.99988 & 0.00012 \\
14 & 99,994 & 0.99994 & 1.99988 & 1.99994 & 0.00006 \\
15 & 99,997 & 0.99997 & 1.99994 & 1.99997 & 0.00003 \\
16 & 99,999 & 0.99999 & 1.99998 & 1.99999 & 0.00001 \\
17 & 100,000 & 1.00000 & 2.00000 & 2.00000 & 0.00000 \\
\hline
\end{tabular}

As I have noticed above, there is a slight difference between the formulations of Zeno's argument with continuous time and discrete time. With continuous time, at each step of Zeno's argument, Achilles has to reach the position occupied by the tortoise. However, with discrete time, Achilles has to reach or overtake the position occupied by the tortoise. This difference results from the fact that the series of the discrete positions of Achilles and the tortoise are not necessarily superimposed. Indeed, the distance $x_{A}\left(t_{k_{n}}\right)$ covered by Achilles during the amount of time $t_{k_{n}}$ is not necessarily a multiple of the elementary distance covered by the tortoise $h v_{T}$. However, this difference between the two formulations of Zeno's argument is not a serious objection. First, one can reformulate both Zeno's arguments with continuous time and discrete time in assuming that, at each step, "Achilles has to overtake the tortoise". In this case, "overtake" means in a broad sense "reach" or "strictly overtake". Second, one should not dismiss the discrete solution of the paradox only because the series of positions of Achilles and the tortoise are superimposed in the continuous case but not in the discrete one. As I shall discuss it in Section 5, despite this difference between both series of positions, they are empirically equivalent.

\subsection{Motion with Discrete Time}

I make clear in what sense Achilles and the tortoise can legitimately be considered in motion although time is represented as discrete. At first glance, a discrete representation of time would indeed be not compatible with motion, as it is exemplified with the Zeno's arrow paradox. 
[S] uppose we consider a period consisting of a thousand instants, and suppose the arrow is in flight throughout this period. At each of the thousand instants, the arrow is where it is, though at the next instant it is somewhere else. It is never moving, but in some miraculous way the change of position has to occur between the instants, that is to say, not at any time whatever. (Russell 1929, in Salmon 2001, p. 50)

Russell assumes here that time is represented as discrete. Under this condition, at each instant $t_{k}$, a moving arrow occupies a unique position $x_{k}$ and, for that reason, cannot be said moving at this instant. Equally, at the next instant $t_{k+1}$, the arrow occupies another unique position $x_{k+1}$. Since there is not any instant between two successive instants $t_{k}$ and $t_{k+1}$, the change of positions cannot be made between them. Therefore, it seems that we have to conclude that the moving arrow never moves when time is represented as discrete. Consequently, if such a conclusion is applied to Achilles and the tortoise, they cannot be said moving.

On contrary to the conclusion of the Arrow paradox, I claim that the arrow, and more generally any body, can be said moving with a discrete representation of time. I indeed endorse the "at-at theory of motion" (Russell 2010, p. 355 and p. 480) $]^{7}$ which applies to a discrete representation of time. According to this account of motion, one cannot say with a single instant if a body moves. One has to compare positions with at least two different instants. As we have seen, position is a function of time within a discrete representation of time. For instance, a particle with constant speed is described by the equation $x_{k}=v t_{k}$. Therefore, a particle can be said in motion because different discrete positions $x_{k}$ correspond to different discrete time $t_{k}$. In summary, a body is said moving if and only if its discrete positions $x_{k}$ are different at different instants $t_{k}$.

This section was dedicated to the discrete treatment of the paradox of Achilles and the tortoise. In the remaining of the paper, I discuss two possible objections (Section 4 and Section 5).

\section{Discrete Classical Mechanics}

First, the discrete solution of the Achilles paradox might be viewed as an ad hoc solution. While the use of a discrete representation of time enables to avoid Zeno's conclusion, it would turn out to be a fruitless representation in general because it could not be used as a framework for physical theories.

This objection is raised because it is generally admitted that it is not possible to formulate classical mechanics without a continuous representation of time. The fundamental laws of motion of classical mechanics are differential equations that require a continuous representation of time. Thus, the laws of motion and the fundamental principles of classical mechanics are lost when time becomes represented as discrete. They are replaced by difference equations but a formulation of classical mechanics grounded on difference equations seems generally impossible. According to Grünbaum, "a physical theory whose fundamental laws take the form of

\footnotetext{
7 See also (Salmon 1980, p. 40-42).
} 
difference equations" is "at best, a gleam in the eyes of hopeful speculative theoreticians"(Grünbaum 1968, in Salmon 2001, p. 246).

On contrary, I claim that classical mechanics can be formulated with a discrete representation of time and, accordingly, the discrete solution of the paradox should not be viewed as an ad hoc solution. There exists nowadays a discrete formulation of classical mechanics, called discrete variational mechanics:

Discrete variational mechanics [is] a formulation of mechanics in discretetime that is based on a discrete analogue of Hamilton's principle, which states that the system takes a trajectory for which the action integral is stationary. $[\ldots]$

Discrete Lagrangian and Hamiltonian mechanics have been developed by reformulating the theorems and the procedures of Lagrangian and Hamiltonian mechanics in a discrete time setting (see, for example, (Marsden et al 2001)). Therefore, discrete mechanics has a parallel structure with the mechanics described in continuous time [...] (Lee et al. 2009, p. 2001 and p. 2004)

In order to better grasp how such discrete formulations are possible, I focus on the case of Discrete Lagrangian Mechanics (DM).

DM has been developed since the 1980's by the 1957 Nobel Laureate in Physics T.D Lee (1983), D'Innocenzo et al. (1987), and Marsden \& West (2001) among others. At the beginning, T.D Lee developed it in order to solve the divergence problems of Quantum Field Theory. He wanted to build up a discrete mechanics conceived as a first step toward fully discrete fundamental theories in which divergences could not occur (Friedberg \& Lee 1983, Lee 1987). Furthermore, this discrete mechanics has been developed as a means to numerically solve classical mechanical systems (Feng 1986, Baez \& Gilliam 1994, Marsden \& Wendlandt 1997). Currently, the studies on this discrete formulation pertain mainly to the fields of computational geometry and mechanics (Stern \& Desbrun 2008, Lee et al. 2009 among others). Numerical computations of mechanical systems turn out to be better - more stable, more accurate - if the discrete equations implemented on computers are not the results of discretizations but are initially derived from discrete variational principles.

Philosophers of science did not yet pay proper attention to such discrete formulations of classical mechanics. This section might thus be a first step toward rectification of this lack of discussions. I begin to introduce the fundamental principles of DM (section 4.1). Then, I sketch how scientists can use DM to describe and predict physical phenomena (section 4.2) before suggesting further current developments within DM (section 4.3).

\subsection{Fundamental Principles}

Discrete Mechanics is based on a discrete least action principle. More precisely, there is a discrete action defined as the $\operatorname{sum} S_{d}=\sum_{k=0}^{N-1} L_{d}\left(t_{k+1}-t_{k}\right)$ where $L_{d}$ is a discrete Lagrangian. It is a function of the discrete coordinates $q_{k}, q_{k+1}$ and the discrete time step $h_{k+1}=t_{k+1}-t_{k}$. This action is the discrete analogue to the traditional action from now called continuous action - defined as the integral of the continuous Lagrangian $S=\int L(q, \dot{q}) d t$. 


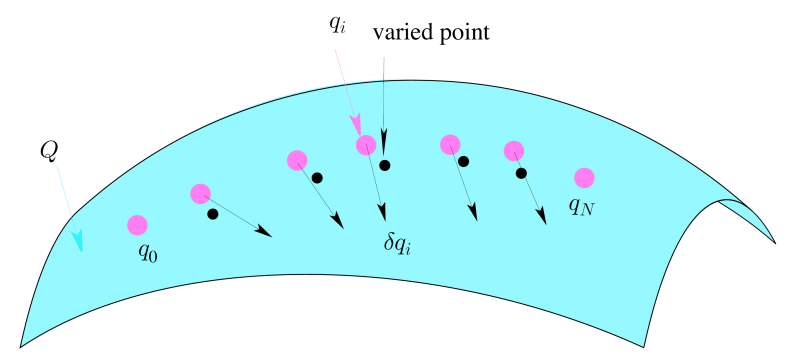

Fig. 1 The discrete form of Hamilton's principle (Lew et al. 2003).

In continuous Lagrangian Mechanics, the Euler-Lagrange equations are deduced from Hamilton's principle according to which the flows of the mechanical systems satisfy $\delta S=0$. In DM, there is also a Hamilton's principle, which states that discrete flows satisfy $\delta S_{d}=0$. This discrete minimization principle leads to the Discrete Euler-Lagrange equations:

$$
h_{k} \frac{\partial L_{d}}{\partial q_{k}}\left(q_{k-1}, q_{k}, h_{k}\right)+h_{k+1} \frac{\partial L_{d}}{\partial q_{k}}\left(q_{k}, q_{k+1}, h_{k+1}\right)=0
$$

These discrete equations are analogous to the continuous Euler-Lagrange equations.

Contrary to the continuous case, another equation results from the discrete minimization principle, which is:

$$
\frac{\partial}{\partial t_{k}}\left(h_{k} \cdot L_{d}\left(q_{k-1}, q_{k}, h_{k}\right)\right)+\frac{\partial}{\partial t_{k}}\left(h_{k+1} L_{d}\left(q_{k}, q_{k+1}, h_{k+1}\right)\right)=0
$$

It corresponds to the conservation of the energy, where the discrete energy is defined as $E_{k}=\frac{\partial}{\partial t_{k}}\left(h_{k} . L_{d}\left(q_{k-1}, q_{k}, h_{k}\right)\right)$. In continuous Lagrangian mechanics, the conservation of energy is already contained in the Euler-Lagrange equations. It can be deduced from them. In DM, it is an independent equation derived from the discrete least action principle ${ }^{8}$

Let us consider, for example, the following discrete Lagrangian: $L_{d}=\frac{1}{2} m v_{k+1}^{2}-$ $V\left(q_{k}\right)$ where $v_{k+1}=\left(q_{k+1}-q_{k}\right) / h_{k+1}$. In these conditions, the first equation corresponds to the discrete motion equation $m \frac{v_{k+1}-v_{k}}{h_{k+1}}=-\frac{\partial V\left(q_{k}\right)}{\partial q_{k}}$. It is a discrete version of the equation of motion of Newton's mechanics. In addition, according to the previous definition of the energy, one has $E_{k+1}=\frac{1}{2} m v_{k+1}^{2}+V\left(q_{k}\right)$. The first term of this

\footnotetext{
8 In continuous mechanics, Euler-Lagrange equations and conservation of energy can also be derived from the least action principle - even if it is not the usual derivation. See for example (Marsden \& West 2001 , p. 467, Chen et al. 2006, p. 227). However, in this case, the latter equation can be deduced from the former. On contrary, this is not possible within DM. This specificity results from the fact that the space coordinate and the time coordinate are treated symmetrically (D'Innocenzo et al. 1987, p. 246). In addition, I would like to emphasize that, as in the continuous case, this framework can be generalized to non-autonomous Lagrangian, i.e time-dependant Lagrangians. In this case, energy is not conserved and the second equation represents the evolution of energy.
} 
equation represents the kinetic energy and the second one the potential energy. The second equation thus states that the discrete total energy is conserved, i.e $E_{k}=E_{k+1}$.

Similarly to the continuous case, there is a discrete Noether theorem within DM that connects laws of conservation to symmetries. In continuous mechanics, if a system is invariant under the infinitesimal transformation $(q, \dot{q}) \rightarrow\left(q^{\varepsilon}, \dot{q}^{\varepsilon}\right)$, Noether theorem states that the following quantity is conserved:

$$
Q=\left.\frac{\partial L}{\partial \dot{q}} \frac{\partial q^{\varepsilon}}{\partial \varepsilon}\right|_{\varepsilon=0}
$$

For example, the linear momentum $Q=m \dot{q}$ is conserved if the system is invariant under spatial translation, i.e if it is invariant under the transformation $q \rightarrow q^{\varepsilon}=q+\varepsilon$. This is for example the case for a free particle. Let us now turn to DM. If a system is invariant under the infinitesimal transformation $\left(q_{k}, q_{k+1}\right) \rightarrow\left(q_{k}^{\varepsilon}, q_{k+1}^{\varepsilon}\right)$, the discrete Noether theorem states that the following quantity is conserved:

$$
Q_{d}=\left.h_{k+1} \frac{\partial L_{d}}{\partial q_{k+1}} \frac{\partial q_{k+1}^{\varepsilon}}{\partial \varepsilon}\right|_{\varepsilon=0}
$$

For example, let us consider the case of a free particle represented by the discrete Lagrangian $L_{d}=\frac{1}{2} m v_{k+1}^{2}$. This system is invariant under spatial translation $q_{k} \rightarrow q_{k}^{\varepsilon}=$ $q_{k}+\varepsilon$. Consequently, the quantity $Q_{d}=m v_{k+1}$ is conserved, which is interpreted as conservation of the linear momentum.

In addition, it can be shown that the discrete flows of DM are symplectic. It means that discrete equations preserve a quantity $\omega$ that can be interpreted geometrically as a volume in the discrete state space ${ }^{9}$ It is similar to the case of continuous mechanics where flows also preserve symplecticity. Finally, I would like to point out that Legendre transformations can also be defined within DM, which enables to connect discrete Lagrangian mechanics with discrete Hamiltonian mechanics (Marsden \& West 2001, p. 486).

\subsection{Exact and numerical solutions}

I would like to make clear how DM can be used in practice by scientists to describe and predict physical phenomena. More precisely, I show that the dynamics of mechanical systems can be studied within DM. First, I emphasize that the equations of DM can be solved analytically for some elementary systems. Second, I discuss the case of the numerical computation of DM equations.

Before investigating the solutions of DM equations, it has to be noticed that there is not a single DM but rather a family of different DM. They depend on the expression of the potential energy $V_{d}$ in the discrete Lagrangian $L_{d}=K_{d}-V_{d}$. The discrete potential energy can be expressed, for example, as $V\left(q_{k}\right), V\left(\left(q_{k+1}+q_{k}\right) / 2\right)$,

\footnotetext{
9 A proper introduction of symplecticity requires tools of differential geometry that are beyond of the scope of this introductory presentation. See for example (Marsden \& West 2001, p. 477).
} 
or $\left(V\left(q_{k+1}\right)+V\left(q_{k}\right)\right) / 2$ among others, where $V(z)$ is the expression for the continuous potential energy 10 Accordingly, the previous section (4.1) describes a general framework for different DM. Currently, several versions of DM are developed. For the purpose of this paper, I focus on the version provided by d'Innocenzo et al. for which $V_{d}=V\left(\left(q_{k+1}+q_{k}\right) / 2\right)$.

First, I would like to point out that several elementary systems can be solved exactly within DM. Therefore, scientists can directly describe the dynamics of such systems with the discrete solutions. In particular, the systems of (i) a free particle, (ii) a particle with constant force - like the gravity force $m g$ - and (iii) the harmonic oscillator, are solved analytically (D'Innocenzo et al. 1987). In a nutshell, the solutions for these systems are:

(i) free particle: $q_{k}=v_{0} t_{k}+q_{0}$

(ii) particle under gravity potential: $q_{k}=\frac{1}{2} g t_{k}^{2}+\left(v_{0}+g h\right) t_{k}+q_{0}$

(iii) harmonic oscillator: $q_{k}=A_{d} \cos \left(\omega_{d} t_{k}\right)+B_{d} \sin \left(\omega_{d} t_{k}\right)$

where $t_{k}=k h$ and $A_{d}, B_{d}, \omega_{d}$ constants that depend on $h$.

I would like to emphasize that, as d'Innocenzo et al. (1987) discuss it, the solution $t_{k}=k h$ for the discrete time series in the case of a free particle is not the only one. There are other solutions for which the discrete instants are $t_{k}=k h_{k}$ where the size of time steps $h_{k}$ is undetermined. ${ }^{11}$ It might be viewed as an issue for the solution of the Achilles paradox since Achilles and the tortoise are represented as two free particles. However, I follow d'Innocenzo et al. who consider such other solutions as irrelevant. Indeed, it is expected that the solution for a free particle corresponds to the solution for a particle with constant force when the force goes to zero. In the case of a particle with non-null constant force, the solution is $t_{k}=k h$. Therefore, the other solutions for a free particle are left out.

Second, when the analytical solutions of DM equations are not known or could not be known, equations can however be solved numerically. In these conditions, scientists could use DM to describe and predict the dynamics of mechanical systems with computers. As I have already emphasized it, DM is nowadays mainly developed by computer scientists in order to make accurate and stable numerical computations possible. The equations of DM can indeed be viewed as variational integrators for continuous mechanics, which are well-known efficient integrators for mechanical systems (Hairer et al. 2006, Keng \& Qin 2010).

In practice, a slight different version of DM is developed by computer scientists. It is more convenient, for numerical purposes, to use a "simplified" version of DM for which the time steps $h_{k}$ are initially chosen to be constant. For example, instead of using the discrete Lagrangian $L_{d}=\frac{1}{2} m v_{k+1}^{2}-V\left(q_{k}\right)$ with $v_{k+1}=\left(q_{k+1}-q_{k}\right) / h_{k+1}$, the velocity is defined as $v_{k+1}=\left(q_{k+1}-q_{k}\right) / h$. This simplification implies that exact

\footnotetext{
10 Furthermore, there are also different versions of DM depending on the initial conditions for the value of the time step. I go back to this point in Section 5.

11 This results from the fact that, in the case of a free particle, the discrete Euler-Lagrange equations and the equation of energy conservation are degenerate. The discrete solution (5) still holds.
} 
conservation of energy may be lost ${ }^{12}$ This is well accepted by computer scientists since energy, even approximatively, is still better preserved than using traditional integrators within continuous mechanics. The equations of the simplified version DM are still symplectic. This property leads to very good approximate conservation of energy (Hairer et al 2006, chap. 1; Stern \& Desbrun 2008) ${ }^{13}$ Therefore, even if the general version of DM - described in section 4.1 - leads to more accurate computations and is theoretically more satisfying, the simplified version of DM is nowadays mainly developed and studied by computer scientists. This can be viewed as a typical scientific practice that consists in simplifying equations for computational purposes. Scientists commonly use simplified physical models if such simplifications lead to much more tractable solutions with sufficiently good approximations.

\subsection{Further developments}

I finish this presentation of DM with current developments that are still in progress. There are generalizations of DM about constrained systems, dissipation, forcing, and collisions. In this section, I briefly describe two of them 14

First, the cases of forcing and dissipation are currently studied within DM. Similarly to continuous Lagrangian mechanics, this generalization requires to extend the least action principle. In continuous mechanics, non-conservative systems are represented by the extended least action principle:

$$
\delta \int_{t_{0}}^{t_{f}} L(q, \dot{q}) d t+\int_{t_{0}}^{t_{f}} f(q, \dot{q}) \cdot \delta q(t) d t=0
$$

where $f$ are non-conservative forces. After integration by parts, this leads to the forced Euler-Lagrange equations. Similarly, for non-conservative systems, there is an extended discrete least action principle with discrete non-conservative forces that are functions of the variables $q_{k}, q_{k+1}$ and $h_{k+1}$. In the same way, after some algebraic manipulations, one is led to the discrete forced Euler-Lagrange equations. Such a generalization thus enables to describe more realistic physical systems within DM.

Second, I would like to point out how collisions can be described within DM. I focus on the case of elastic collisions even if more complex collisions can be treated. My aim here is to answer to a possible objection against DM. Let us consider the case of a collision between two free particles. A point particle $A$ moves at constant velocity $v_{A}$ towards another particle $B$. Its positions are $x_{A}\left(t_{k}\right)=v_{A} t_{k}$ with $t_{k}=k h$.

\footnotetext{
12 The fact that time step $h_{k}$ is allowed to vary within DM guarantees that autonomous systems exactly preserve energy, momenta and symplecticity, as in continuous mechanics. However, as we have seen in the examples above, it does not mean that discrete time step must vary. Even if time step is allowed to vary in the discrete least action principle, the latter can lead to constant discrete time step depending on mechanical systems and versions of DM.

13 See (Kane et al. 1999) for a comparison between the "simplified" version and the "general" version of DM.

14 Such cases are currently developed in the context of numerical computation. As it should be clear from Section 4.2, many generalizations thus pertain to the "simplified" version of DM. However, this restriction is not mandatory. Generalizations for the general version of DM are also currently developed as it is noticed in (Lee et al. 2009, p. 2017) and started to be achieved in (Pekarek 2010, chapter 3).
} 
The particle $B$ is at rest in $x_{B}=\tilde{x}$ such as $v_{A}(k-1) h<\tilde{x}<v_{A} k h$. In other words, $B$ is "between" the two successive positions of the $A$ particle, $x_{A}\left(t_{k-1}\right)$ and $x_{A}\left(t_{k}\right)$. In these conditions, it seems that $A$ cannot collide with $B$ since there are not any positions of $A$ that correspond to the position of $B$. This would be a serious objection against the viability of DM.

On contrary, I argue that $A$ can collide with $B$. This claim is supported by recent developments on collisions within DM (Fetecau et al. 2003, Pekarek \& Marsden 2008, Pekarek 2010). In continuous Lagrangian mechanics, elastic collisions are treated in minimizing the action under extra constraints. The variations of the position $\delta q$ are taken equal to zero at the impact point $\tilde{q}$, which occurs at the instant $\tilde{t}$. This leads to the usual Euler-Lagrange equations and to two extra equations corresponding to the conservation of energy and linear momentum at the impact. In DM, elastic collisions are also treated in minimizing the discrete action under extra constraints. The variations of the discrete positions $\delta q_{k}$ and the discrete time $\delta t_{k}$ are taken equal to zero at the impact point $\tilde{q}$, which occurs at the instant $\tilde{t}$. This minimization also leads to the usual discrete Euler-Lagrange equations and extra discrete equations. Before and after the impact, the system reduces to the case of a free particle. As we have seen in Section 4.2, the discrete time step for free particle is constant and the position of $A$ is given by the formula $x_{A}\left(t_{k}\right)=v_{A} k h$. However, the extra equations imply that the time step and the discrete elementary distance are not constant near the impact point. More precisely, the coordinate $(\tilde{q}, \tilde{t})$ is interpolated in the discrete sequence $\left\{\left(q_{0}, t_{0}=0\right),\left(q_{1}, t_{1}=h, \ldots,\left(q_{k-1}, t_{k-1}=(k-1) h\right),\left(q_{k}, t_{k}=k h\right), \ldots\left(q_{N}, t_{N}=N h\right)\right\}\right.$. In other words, there is an extra discrete position $\tilde{q}$ between $q_{k-1}$ and $q_{k}$ at the extra instant $\tilde{t}=(k-\alpha) h$ where $\alpha \in[0,1]$. Therefore, the collision of the free particle $A$ occurs exactly at $x_{B}=\tilde{x}$.

Discrete mechanics did not yet exist when Grünbaum doubted that it was possible to formulate classical mechanics with difference equations. A discrete version of classical mechanics was legitimately thought as very speculative. But nowadays, with the development of discrete variational mechanics, it seems difficult to maintain such a claim. I recognize that works on DM are still in progress. However, in my view, DM is sufficiently well developed to dismiss the objection that it is only a speculative hypothesis. Therefore, the discrete solution for the paradox of Achilles and the tortoise cannot be considered as an ad hoc solution. It is based on a discrete representation of time that can also be used to describe and predict physical phenomena.

\section{Empirical Equivalence}

The second possible objection against the discrete solution of the paradox of Achilles and the tortoise that I answer is the following one. One could argue that the discrete solution of the paradox might be falsified. If an experiment is set up with the aim to follow Zeno's argument, one could expect that the series of the discrete positions of Achilles would be in conflict with empirical data. In this section, I first argue that the series of the discrete and continuous positions of Achilles cannot be distinguished by any experiment (section 5.1). Then, I defend that, more generally, DM and continuous mechanics are empirically equivalent (section 5.2). 
First of all, I would like to emphasize the discrete solution for the paradox of Achilles holds as far as physical phenomena are described in a classical way. As it should be clear from Section 4, the use of a discrete representation is embedded in a discrete formulation of classical mechanics. Therefore, by experiment, I mean in this section experiment in a classical context.

\subsection{Continuous and Discrete Achilles Races}

Let us suppose that one tries to determine experimentally the positions of Achilles following Zeno's argument. Such an experiment would certainly not be feasible in practice. The distances between Achilles and the tortoise become too small to be measured. But let us imagine an idealized two mass points system representing Achilles and the tortoise with the features of the footrace. In addition, let us assume that there is an ideal device enabling to measure the series of the positions of the two mass points with a finite accuracy $\varepsilon_{\exp }$ as small as we want. Under these conditions, I claim that we cannot reject the use of a discrete representation of time on the grounds that it would be in conflict with any experiment. Zeno's argument within a discrete and a continuous time are empirically equivalent.

The reason of the empirical equivalence is the following one. There are as many discrete representations of time as values for the discrete time step $h$. For example, there is a discrete representation of time which corresponds to the discrete time step $h=10^{-5}$. Another representation of time corresponds to a discrete time step $h=$ $10^{-15}$ and so on. Consequently, for any value of the accuracy $\varepsilon_{\text {exp }}$, it always exists a discrete representation of time for which the series of the positions and instants of the two mass points within discrete time and continuous time cannot be distinguished by a measurement.

For example, let us consider again the discrete representation of time with the discrete time step $h=10^{-5}$. We have seen that Achilles overtakes the tortoise after 17 steps of Zeno's argument (Section 3). Let us compare, on the one hand, the differences between the series of the instants of Zeno's argument and, on the other hand, the differences between the series of positions of Achilles. One can show that the maximal differences occur at the $11^{\text {th }}$ step. They are respectively $8.28125 \times 10^{-6}$ for the instants and $1.65625 \times 10^{-5}$ for the positions. Therefore, for devices that cannot provide an accuracy better than, for example, $2 \times 10^{-5}$ for positions and $10^{-5}$ for durations, Zeno's argument within continuous and this discrete representation of time are indistinguishable. More generally, it is proved that the differences with a continuous time and a discrete time are never greater than $\varepsilon_{1}=h v_{A} /\left(1-v_{T} / v_{A}\right)$ for the positions of Achilles and $\varepsilon_{2}=h /\left(1-v_{T} / v_{A}\right)$ for the instants of Zeno's argument.

The parameters $\varepsilon_{1}$ and $\varepsilon_{2}$ vary proportionally with the discrete time step $h$. Thus, for any given velocities of Achilles and the tortoise, if the discrete time step $h$ is taken sufficiently small, the parameters become as small as we want. Consequently, whatever the accuracy of the device, there exists always a discrete representations of time that makes Zeno's arguments indistinguishable. More precisely, for any given accuracy $\varepsilon_{\text {exp }}$, it suffices to choose a discrete representation satisfying $h<\left(\varepsilon_{\exp } / v_{A}\right)(1-$ 
$\left.v_{T} / v_{A}\right)$. In this case, the positions of Achilles with continuous time and discrete time are empirically indistinguishable.

One might object that such an idealized experiment is not compatible with the laws of physics, in particular the Heisenberg uncertainty principles of quantum mechanics. This kind of objection is provided by Alper \& Bridger (1997) against McLaughin \& Miller's solution (1992). It seems to equally apply to the present discrete solution. According to quantum-theoretic limitations on measurement, it would be impossible to know with sufficient accuracy the position of a particle by following Zeno's argument after several steps. The use of a discrete representation of time to solve the paradox would thus be futile. I answer to this objection by emphasizing that the discrete solution provided in this paper only pertains to classical physics. It is based on a discrete formulation of classical mechanics for which there are no uncertainty principles. As far as one investigates the Achilles race within classical mechanics, the idealized experiment described in this section is compatible with the laws of physics ${ }^{15}$

However, there might be a case for which the continuous series and the discrete one would be distinguishable. Let us consider that the velocity of Achilles goes to infinity. Since empirical indistinguishability is guaranteed by the relation $h<\left(\varepsilon_{\exp } / v_{A}\right)\left(1-v_{T} / v_{A}\right)$, the discrete time step would have to be equal to zero. Therefore, if the velocity of Achilles tends to infinity, the empirical equivalence of both series would not be guaranteed. First of all, I point out that it does not follow that both series would be distinguished but only that empirical equivalence would not be guaranteed. Maybe a stronger constraint on $h$ can be established that, for example, would not depend on the velocity of Achilles. In any case, it does not seem a major objection since assuming an unbounded velocity for Achilles might not be compatible with the usual (continuous) formulation of the paradox of Achilles and the tortoise. According to the usual formulation of the paradox, the relative position of Achilles to the tortoise is $x_{A / T}=-x_{0}\left(v_{T} / v_{A}\right)^{n}$ (Section 2). If the velocity of Achilles tends to infinity, the relative distance of Achilles tends to zero even if the number $n$ of steps of Zeno's argument is bounded. In my view, the paradox of Achilles and the tortoise requires that the velocity of Achilles and the tortoise are non-null and bounded. The empirical equivalence between the continuous and the discrete Achilles races holds if one takes initially bounded velocities for Achilles and the tortoise and a bounded accuracy $\varepsilon_{\text {exp }}$. The value of the discrete time step is allowed to vary afterwards.

\subsection{Discrete Mechanics and Empirical Equivalence}

Even if the series of the discrete and continuous positions of Achilles cannot be experimentally distinguished, one can however suppose that DM could be in conflict with empirical data about other mechanical systems. In this case, it would undermine the viability of DM and the relevance of the discrete solution of the paradox. In this

\footnotetext{
15 If we want to discuss the consequences of quantum limitations on the Achilles paradox, one should investigate a discrete formulation of quantum mechanics. I suggest in the conclusion some perspectives in this direction.
} 
section, I answer to this objection. I defend that DM is empirically adequate as continuous classical mechanics is. In other words, DM and continuous mechanics would be empirical equivalent.

The main argument in favour of the empirical equivalence is based on the property that the equations of DM tend to the equations of continuous mechanics when the discrete time step goes to zero:

Obviously, physical quantities [of DM] must tend to corresponding continuous classical variables when the discrete time intervals tend to zero. (D'Innocenzo et al. 1987 , p. 246)

More precisely, the discrete action $S_{d}$ is an approximation of the continuous action $S$ such as it tends to the latter when the discrete time step goes to zero ${ }^{16}$ Thus, the discrete Euler-Lagrange equations, the equation of the conservation of energy, and their respective solutions tend to the continuous case. In these conditions, one can assume that, if the discrete time step is taken sufficiently small, the solutions of the DM equations cannot be distinguished from the equations of continuous mechanics.

To my knowledge, there is no theorem that could guarantee that, in all cases, the empirical equivalence holds. Nevertheless, I do not know any counter-examples against the empirical equivalence. In order to support my claim, I investigate two cases that exemplify the empirical equivalence.

First, let us consider the case of the harmonic oscillator provided in Section 4.2. If one takes a small time step $h$, the relative difference between the discrete frequency $\omega_{d}$ and the continuous one $\omega_{0}$ is : $\left(\omega_{0}-\omega_{d}\right) / \omega_{0}=\left(\omega_{0}^{2} h^{2}\right) / 12\left[{ }^{17}\right.$ For example, with an unit continuous frequency, if $h=10^{-10}$, the order of magnitude of the relative difference is $10^{-21}$. Therefore, if $h$ is taken sufficiently small, it is not possible to distinguish the frequencies predicted within continuous and discrete mechanics. In addition, let us define $q(t)$ and $q_{k}$ the solutions of the equations with continuous and discrete mechanics. Let us also introduce the relative position $\sigma\left(t_{k}\right)=\left|\left(q\left(t_{k}\right)-q_{k}\right) / q_{0}\right|$, which measures the relative difference between both solutions. In this case, on can check that $\sigma\left(t_{k}\right)$ is smaller than $\omega_{0}^{3} h^{2} t_{k} / 12 \sqrt{18}$ Therefore, if the best accuracy for the measures of position is $\varepsilon$, the positions of the harmonic oscillator within continuous and discrete mechanics cannot be distinguished if $h<\sqrt{\frac{12 \varepsilon}{\omega_{0}^{3} T}}$, where $T$ is the time interval of measurement ${ }^{19}$ Therefore, one can legitimately suppose that, for $h$ sufficiently small, the models of the harmonic oscillator with continuous and discrete mechanics are empirically equivalent. More generally, when a system is not sensitive

\footnotetext{
16 Since the time step is allowed to vary, $S_{d}$ tends to $S$ when the initial time step $h_{0}$ and the initial position step $\left(q_{1}-q_{0}\right)$ tend to zero. For the sake of the argument, I talk about time step $h$ even if one has to keep in mind that the parameters that go to zero are $h_{0}$ and $\left(q_{1}-q_{0}\right)$.

17 See (D'Innocenzo et al. 1987, p. 250).

18 For simplifications, I have used the initial conditions such as the continuous and the discrete solutions are $q(t)=q_{0} \cos \left(\omega_{0} t\right)$ and $q_{k}=q_{0} \cos \left(\omega_{d} t_{k}\right)$.

19 According to the model of the harmonic oscillator, $\omega_{0}^{2}=k / m$, where $k$ is the constant of the spring and $m$ the mass of the body. There might be no empirical equivalence if $k$ tends to infinity and if $m$ tends to zero. However, such extreme cases might not be compatible with the representation of a physical system by a harmonic oscillator. For example, nonlinearity would have to be taken into account. In any case, as far as $k$ and $m$ are non-null and bounded, the empirical equivalence holds. Equally, the empirical equivalence requires that $T$ is bounded. One can take, for example, the order of magnitude of the age of the universe.
} 
to initial conditions - like the harmonic oscillator - one can expect that empirical equivalence holds. Since a small difference in the initial conditions for such systems does not imply to big differences in the solutions, one can assume that a discrete treatment does not imply large differences if the time step is taken sufficiently small.

Second, I would like to discuss what would be the worst case against the empirical equivalence thesis. Let us consider the case of systems sensitive to initial conditions within continuous mechanics. It is known that the discretization of chaotic differential equations can suppress the chaotic behaviour of the system (Corless at al. 1991) ${ }^{20}$ In other words, the system described with differential equations has not the same general behaviour than the one described with difference equations. Accordingly, they are not empirically equivalent. In these conditions, it is questionable how chaotic systems are treated within DM and whether empirical equivalence holds. As far I know, there are not many discussions of such systems. However, there is at least one study in (Rowley \& Marsden 2002, Lew et al. 2003). It is the case of a system of four interacting vortices. In using a "simplified" version of DM, the authors investigate the Poincaré sections of the system. They show that DM leads to chaotic behaviour if the time step is taken sufficiently small ${ }^{21}$ This property is not so surprising. Corless et al. (1991) emphasize that the suppression of chaotic behaviour due to discretization occurs if time steps are taken too large. However, what was not expected is that the Poincaré sections are actually more reliable within DM rather within continuous mechanics in using traditional numerical integrators (Rowley \& Marsden 2002). Further discussions on chaotic systems within DM should continue but, according to this study, one can legitimately assume that the empirical equivalence holds if the time step is taken sufficiently small.

\section{Conclusion}

The aim of this paper was to show that the paradox of Achilles and the tortoise can be solved if time is represented as discrete. More precisely, I have argued that in replacing the continuous parameter $t$ of classical mechanics by a discrete parameter $t_{k}$, Achilles overtakes the tortoise in a finite number of steps of Zeno's argument. Thus, one has not to endorse that Achilles performs supertasks to solve the paradox but rather to change the way that time is represented.

This discrete solution crucially depends on whether the discrete representation of time is acceptable. Accordingly, I have first made clear that the resulting notion of motion can be conceived within the "at-at" theory. Then, I have argued that the discrete solution is embedded in a new formulation of classical mechanics, called Discrete Mechanics. Finally, I have rejected that the discrete solution could be falsified by an experiment in a classical context. Whatever the accuracy of measurements, it is always possible to find a discrete representation of time - i.e a version of DM

\footnotetext{
${ }^{20}$ Equally, it is well known that the discretization of non-chaotic differential equations can lead to discrete chaotic behaviour, like the logistic equation. However, the discrete and the continuous solutions share the same behaviour if the time step is sufficiently small, i.e smaller than a critical value.

${ }^{21}$ More precisely, they use the discrete Lagrangian $L_{d}\left(q_{k}, q_{k+1}\right)=L\left(\left(q_{k+1}+q_{k}\right) / 2,\left(q_{k+1}-q_{k}\right) / 2\right)$.
} 
- that makes the series of positions of Achilles with continuous and discrete time indistinguishable.

In my view, the acceptability of the discrete solution of Achilles paradox mainly depends on the viability of DM. Our intuitions on motion have indeed to be guided by the way that physical theories represent time and moving bodies. I defend that discrete mechanics is a genuine framework for physical theories based on a discrete representation of time. In order to fulfil this goal, I have described its first principles and presented how it can be used to describe physical phenomena. I have also argued that DM is as empirically adequate as continuous classical mechanics.

This discussion might be a first step towards further investigations on DM since little attention has been paid to DM yet. First, the question whether discrete mechanics is an autonomous physical theory or a discrete formulation of classical mechanics, and its philosophical implications should be investigated. Second, as we have seen, there is not a single discrete mechanics but a family of many discrete mechanics depending on the value of the discrete time step and, also, on the way that discrete Lagrangians are defined. Thus, the question of the unity of DM should also be tackled. Third, one might investigate whether DM is preferable to continuous traditional mechanics. Even if DM might be a genuine discrete physical theory, continuous mechanics could be preferable to scientists because, for example, it would be simpler.

Finally, I would like to point out that there are discrete variational formulations of other physical theories. They should be investigated from a philosophical point of view. In his seminal papers, T.D. Lee develops discrete formulations for quantum mechanics and relativistic field theory (Lee 1983, Friedberg \& Lee 1983, Lee 1987). They have lead to some discussions in (Bracci et al. 1983, D'Innocenzo et al. 1984). Currently, variational discrete formulations of hydrodynamics and electromagnetism are being developed in a computational context (Gawlik et al. 2011, Pavlov et al. 2011, Desbrun et al. 2014, Stern et al. 2014). Thus, the discrete solution of the paradox of Achilles presented in this paper is part of a more general project, which is the study of the philosophical implications of discrete formulations of physical theories.

Acknowledgements

\section{References}

Alper, J. S. \& Bridger M. (1997). Mathematics, Models, and Zeno’s Paradoxes of Motion. Synthese, 110, 143-166.

Alper, J.S. \& Bridger, M. (1998). Newtonian Supertasks: A Critical Analysis. Synthese, 114 (2), 355-369. Baez, J.C. \& Gilliam, J.W. (1994). On Algebraic Approach to Discrete Mechanics. Letters in Mathematical Physics, 31, 205-212.

Benacerraf, P. (1962). Tasks, Super-Tasks, and the Modern Eleatics. The Journal of Philosophy, 59 (24), 765-784.

Black, M. (1951). Achilles and the Tortoise. Analysis, 11(5), 91-101.

Bracci, L., Fiorentini, G., Mezzorani, G., Quarati, P. (1983). Bounds on a Hypothetical Fundamental Length. Physics Letters, 133B (3-4), 231-233.

Burke, M. B. (2000). The Impossibility of Superfeats. The Southern Journal of Philosophy, 38, 207-220. Carnap, R. (1966). Philosophical Foundations of Physics. New York and London: Basic Books.

Chen, J.B., Guo, H.Y., Wu, K. (2006). Discrete Total Variation Calculus and Lee's Discrete Mechanics. Applied Mathematics and Computation, 177, 226-234. 
Chihara, C. (1965). On the Possibility of Completing an Infinite Task. Philosophical Review, 74, 74-87. Corless, R. M., Essex, C., Nerenberg, M.A.H. (1991). Numerical Methods Can Suppress Chaos, Physics Letters A, 157 (1), 27-36.

D'Innocenzo, A., Renna, L., Rotelli, P. (1987). The Oscillator in Discrete Mechanics, Physics Letters, 145B (3-4), 268-270.

D'Innocenzo, A., Renna, L. \& Rotelli, P. (1987). Some Studies in Discrete Mechanics. European Journal of Physics, 8, 245-252.

Desbrun, M., Gawlik, E., Gay-Balmaz, F., Zeitlin, V. (2014). Variational Discretization for Rotating Stratified Fluids. AIMS' Journals, 34 (2).

Feng, K. (1986). Difference Schemes for Hamiltonian Formalism and Symplectic Geometry. Journal of Computational Mathematics, 4, 279-289.

Feng, K. \& Qin, M. (2010). Symplectic Geometric Algorithms for Hamiltonian Systems. Heidelberg, Dordrecht, London, New-York : Springer.

Fetecau, R. C., Marsden, J. E., Ortiz, M. \& West, M. (2003). Nonsmooth Lagrangian Mechanics and Variational Collision Integrators. SIAM Journal of Applied Dynamical Systems, 2 (3), 381-416.

Forrest, P. (1995). Is Space-Time Discrete or Continuous? An Empirical Question. Synthese, 103 (3), 327-354.

Friedberg, R., Lee, T. D. (1983). Discrete Quantum Mechanics. Nuclear Physics, B225, [FS9], 1-52.

Gawlik, E., Mullen, P., Pavlov, D., Marsden, J.E, \& Desbrun, M. Geometric, Variational Discretization of Continuum Theories. Physica D: Nonlinear Phenomena, 240 (21), 1724-1760.

Grünbaum, A. (1968). Modern Science and Zeno's Paradoxes. London: George Allen and Unwin Ltd, chapter 2.

Grünbaum, A. (1969). Can an Infinitude of Operations Be Performed in a Finite Time?. The British Journal for the Philosophy of Science, 20(2), 203-218.

Hairer, E., Lubich, C. \& Wanner, G. (2006). Geometric Numerical Integration: Structure-Preserving Algorithms for Ordinary Differential Equations. Berlin, Heidelberg: Springer.

Laraudogoitia, J. P. (2006). A Look at the Staccato Run. Synthese, 148, 433-441.

Lee, T. D. (1983). Can Time Be a Discrete Dynamical Variable?. Physics Letters, 122B (3-4), 217-220. Lee, T. D. (1987). Difference Equations and Conservation Laws. Journal of Statistical Physics, 46 (5-6), 843-860.

Lee, T., Leok, M. \& McClamroch, H. (2009). Discrete Control Systems. In Robert A. Meyer (Ed.). Encyclopedia of Complexity and Systems Science. New York: Springer, 2002-2019.

Lew, A., Marsden J.E., Ortiz M., West, M. (2003). An Overview of Variational Integrators. In Franca, L.P. (Ed.) Finite Element Methods : 1970's and Beyond, Barcelona: CIMNE, 1-18.

Marsden, J. E. \& Wendlandt, J.M. (1997). Mechanical Systems with Symmetry, Variational Principles and Integration Algorithms. In Alber, M. et al. (Eds) Current and Future Directions in Applied Mathematics, Boston : Birkhäuser, 219-261.

Marsden, J. E. \& West M. (2001). Discrete Mechanics and Variational Integrators. Acta Numerica, 10, 357-514.

McLaughlin, W. I., \& Miller, S. L. (1992). An Epistemological Use of Nonstandard Analysis to Answer Zeno's Objections against Motion. Synthese, 92, 371-384.

McLaughlin, W. I. (1998). Thomson's Lamp is Dysfunctional. Synthese, 116, 281-301.

Newton-Smith, W. H. (1980). The Structure of Time. London: Routledge \& Kegan Paul.

Pavlov, D., Mullen, P., Tong Y., Kanso, E. , Marsden, J.E. \& Desbrun M. (2011). Structure-Preserving Discretization of Incompressible Fluids, Physica D : Nonlinear Phenomena, 240, 6 (1), 443-458.

Pekarek, D.N., Marsden, J.E. (2008). Variational Collision Integrators and Optimal Control. In Proc. of the 18th International Symposium on Mathematical Theory of Networks and Systems.

Pekarek, D.N. (2010). Variational Methods for Control and Design of Bipedal Robot Models. PhD Thesis.

Caltech.http://thesis.library.caltech.edu/5878/2/Pekarek_CIT_Thesis.pdf

Rowley, C. W. \& J.E. Marsden (2002). Variational Integrators for Point Vortices, Proc. CDC, 40, 15211527.

Russell, B. (1929). Our Knowledge of the External World. New York: Norton \& Company, lecture 6, $182-198$.

Russell, B. (2010). The Principles of Mathematics. London \& New York: Routledge Classics.

Salmon, W. (1980). Space, Time and Motion: A Philosophical Introduction. Minneapolis: University of Minnesota Press.

Salmon, W. (Ed.) (2001). Zeno's Paradoxes. Indianapolis, Cambridge: Hackett.

Stern, A. \& Desbrun, M. (2008). Discrete Geometric Mechanics for Variational Time Integrators. In Discrete Differential Geometry : An Applied Introduction, Siggraph 2006 Course Notes, chap. 15. 
Stern, A., Y. Tong, M. Desbrun, and J. E. Marsden (2014). Geometric Computational Electrodynamics with Variational Integrators and Discrete Differential Forms. Geometry, mechanics, and dynamics: the legacy of Jerry Marsden, Fields Inst. Commun., Springer. In press. preprint: http://arxiv. org/abs/ 0707.4470

Thomson, J., (1970). Comments on Professor Benacerraf's Paper, in Salmon (2001), 130-138.

Van Bendegem, J. P. (1987). Zeno's Paradoxes and the Tile Argument. Philosophy of Science, 154(2), 295-302.

Van Bendegem, J. P. (1995). In Defence of Discrete Space and Time. Logique \& Analyse, 38, 127-150.

Wisdom, J.O. (1952). Achilles on a Physical Racecourse. Analysis, 12(3), 67-72.

Whitehead, A.N. (1979), Process and Reality, New York: The Free Press.

Whitehead, A.N. (2000), The Concept of Nature, Cambridge: Cambridge University Press.

Whitrow, G. J. (1980). The Natural Philosophy of Time. Oxford: Oxford University Press. 